\title{
How to Characterize 3D Microstructure Formation from Micro- to Atomic Scale for the Example of Al-Si Cast Alloys
}

\author{
Frank Mücklich $^{1,2}$, Jenifer Barrirero ${ }^{1}$, Anastasia Kruglova ${ }^{1}$ and Michael Engstler ${ }^{1,2}$ \\ ${ }^{1 .}$ Functional Materials, Department Material Science and Engineering, Saarland University, \\ Saarbrücken, Germany \\ 2. Material Engineering Center Saarland (MECS), Steinbeis Research Center, Saarbrücken, Germany
}

\begin{abstract}
Aluminum foundry alloys with Silicon as the major alloying element are widely used in industry due to their excellent mechanical properties, castability and low density. The AlSi system forms a complex eutectic phase at $12 \mathrm{wt} \%$ silicon. The brittle eutectic silicon has a plate like morphology and forms an interconnected network which lowers the ultimate strain of these alloys. The addition of small amounts of strontium in the range of 150ppm changes the morphology of the eutectic towards a fine, coral like structure and thus enhances the mechanical properties [1]. This so called modification is widely used in industry but still not well understood. Heterogeneous modification still lowers the ultimate strain and the alloy cannot be used at its full potential.

One possible explanation for the change in growth of the eutectic silicon is the so called impurity induced twinning theory (IIT) [2]. Strontium atoms are adsorbed onto growth steps of the solid-liquid interface of eutectic silicon leading to a higher twinning probability. Thus the eutectic silicon starts branching and the morphology is changed from plate like to coral like.
\end{abstract}

High purity Al-7wt\%Si alloys in the unmodified and modified (150ppm Sr) condition were used in this study. The morphology of the eutectic phase was analyzed by means of FIB/SEM serial sectioning tomography on a FEI Helios Nanolab 600. The unmodified alloy shows a plate like morphology (Figure 1a). Different particles are shown in different colors. As nearly the whole volume has the same color, most of the silicon plates are interconnected. Only particles at the border are not connected but they may be outside the studied volume. The modified eutectic (Figure 1b) has a coral like morphology with separated colonies. EBSD measurements show, that these colonies have different orientations.

To study the effect of different morphologies on the mechanical properties a virtual microstructure design approach was used [3]. By using different algorithms the coral like morphology can be modeled (Figure 1c) and used in finite element simulations to predict mechanical properties. By changing parameters of the modeling slightly different structures are obtained and an ideal structure in the sense of high mechanical properties can be found.

The role of strontium during modification was analyzed using laser pulsed atom probe tomography. Samples were prepared by means of FIB target preparation on a FEI Helios Nanolab 600. Atom probe tomography was done using a Cameca LEAP 3000 X HR system. In the modified samples different strontium rich structures were found (Figure 2) [4]. These findings support the impurity induced twinning theory although the role of aluminum segregations inside the eutectic silicon still remain unclear. First results comparing TEM studies and results from atom probe tomography will be presented.

The example of Al-Si cast alloys impressively shows, that the combination of different tomographic techniques at different length scales with microstructure modeling and simulation can lead to a better understanding of microstructure formation and its influence on the materials mechanical properties [5]. 


\section{References:}

[1] J E Gruzleski, B M Closset, The treatment of liquid aluminium-silicon alloys, American foundrymen's society, Illinois, 1990

[2] S Lu, A Hellawell, Metall. Trans. A 18 (1987) p. 1721.

[3] G Gaiselmann et al, Computational Materials Science 69 (2013) p. 289

[4] J Barrirero et al in ,3D Microstructure Meeting“, ed. F. Mücklich et al, (MatInfo, Frankfurt) p. 80 [5] The authors acknowledge the collaboration with O. Stenzel, G. Gaiselmann and. V. Schmidt, Institute of Stochastics, Ulm University,

a)

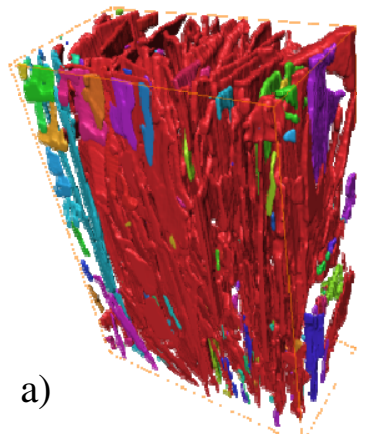

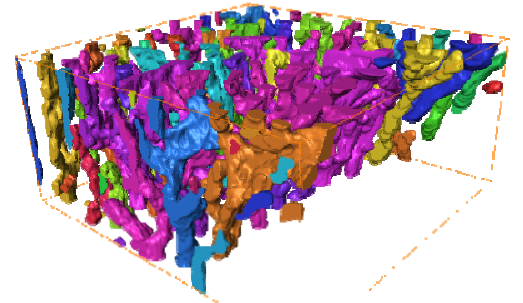

b)

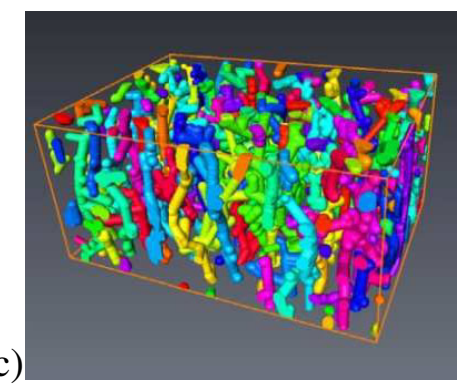

Figure 1. a) Reconstruction from FIB/SEM tomography of the eutectic silicon in an unmodified AlSi7 alloy, approx. 70x100x40 $\mu \mathrm{m}^{3}$, b) Reconstruction of an modified AlSi7 alloy, approx. 37x 17x35 $\mu \mathrm{m}^{3}, \mathrm{c}$ ) Simulation of the coral-like structure of an modified AlSi7 alloy. [3]

a)

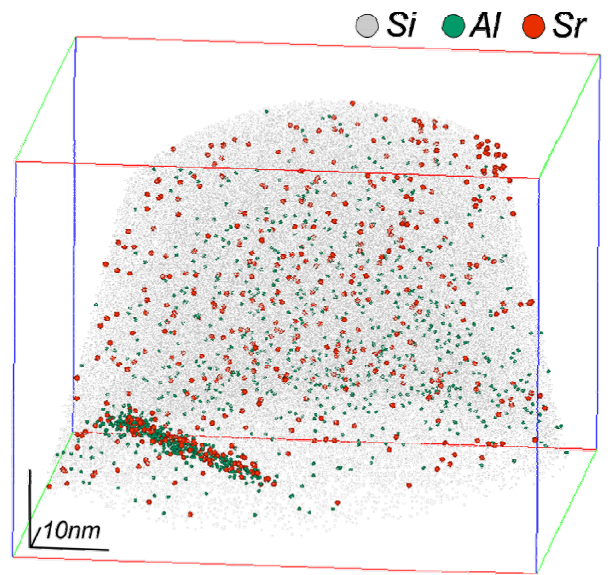

b)

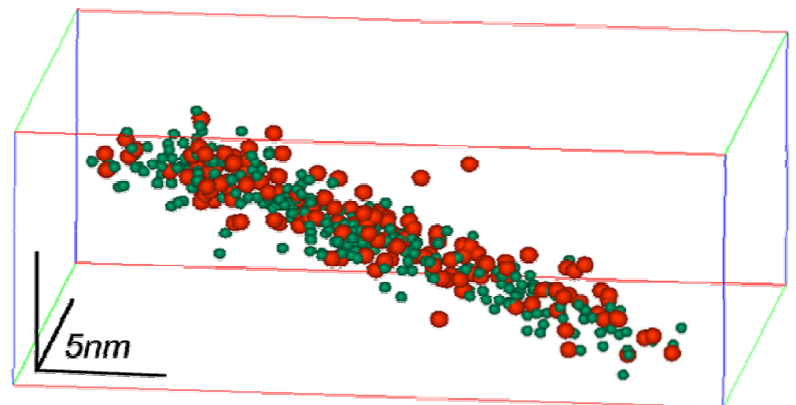

Figure 2. a) Reconstruction from atom probe tomography of the eutectic silicon of a modified AlSi7 alloy. b) Magnification of a $\mathrm{Sr}$ and $\mathrm{Al}$ rich precipitate. 